\title{
Neighborhood-Level Poverty at Menarche and Prepregnancy Obesity in African-American Women
}

\author{
Andrea E. Cassidy-Bushrow, ${ }^{1}$ Rosalind M. Peters, ${ }^{2}$ Charlotte Burmeister, ${ }^{1}$ \\ Lawrence F. Bielak, ${ }^{3}$ and Dayna A. Johnson ${ }^{1,3,4}$ \\ ${ }^{1}$ Department of Public Health Sciences, Henry Ford Hospital, One Ford Place, Detroit, MI 48202, USA \\ ${ }^{2}$ College of Nursing, Wayne State University, 5557 Cass Avenue, Detroit, MI 48202, USA \\ ${ }^{3}$ Department of Epidemiology, University of Michigan, 1415 Washington Heights, Ann Arbor, MI 48109, USA \\ ${ }^{4}$ Division of Sleep and Circadian Disorders, Brigham and Women's Hospital and Harvard Medical School, \\ 221 Longwood Avenue, Boston, MA 02115, USA
}

Correspondence should be addressed to Andrea E. Cassidy-Bushrow; acassid1@hfhs.org

Received 29 February 2016; Revised 11 May 2016; Accepted 25 May 2016

Academic Editor: Debbie Smith

Copyright (C) 2016 Andrea E. Cassidy-Bushrow et al. This is an open access article distributed under the Creative Commons Attribution License, which permits unrestricted use, distribution, and reproduction in any medium, provided the original work is properly cited.

\begin{abstract}
Introduction. Menarche is a critical time point in a woman's reproductive system development; exposures at menarche may influence maternal health. Living in a poorer neighborhood is associated with adult obesity; however, little is known if neighborhood factors at menarche are associated with prepregnancy obesity. Methods. We examined the association of neighborhood-level poverty at menarche with prepregnancy body mass index category in 144 pregnant African-American women. Address at menarche was geocoded to census tract (closest to year of menarche); neighborhood-level poverty was defined as the proportion of residents living under the federal poverty level. Cumulative logistic regression was used to examine the association of neighborhood-level poverty at menarche, in quartiles, with categorical prepregnancy BMI. Results. Before pregnancy, 59 (41\%) women were obese. Compared to women in the lowest neighborhood-level poverty quartile, women in the highest quartile had 2.9 [1.2, 6.9] times higher odds of prepregnancy obesity; this was slightly attenuated after adjusting for age, marital status, education, and parity (odds ratio: $2.3[0.9,6.3])$. Conclusions. Living in a higher poverty neighborhood at menarche is associated with prepregnancy obesity in African-American women. Future studies are needed to better understand the role of exposures in menarche on health in pregnancy.
\end{abstract}

\section{Introduction}

Prepregnancy obesity is associated with adverse pregnancy and birth outcomes, including increased risk of gestational diabetes, preeclampsia, caesarean section, and having a largefor-gestational-age neonate $[1,2]$. Prepregnancy obesity rates in the US are high, affecting approximately 1 in 5 pregnancies [3]. African-American women have increased risk of prepregnancy obesity [4]. There is growing interest in intervening on weight during the preconception phase to improve perinatal outcomes [5]. Importantly, intervening earlier, such as during puberty, may have a greater impact on preventing or reducing the burden of obesity in pregnancy as child and adolescent obesity is strongly associated with adulthood obesity [6].
There is limited, but growing, evidence regarding the role of childhood factors and experiences with obesity during pregnancy [7, 8]. Menarche is a critical time point in a woman's sexual development, reflecting significant biological changes and signaling the transition to the reproductive phase [9]. Obesity in childhood is associated with early age of menarche [10]. The association between obesity at menarche and adult obesity, however, is not fully explained by childhood obesity nor with age at menarche [11, 12]. Age at menarche may be a proxy for sexual maturation reflecting the influence of other factors, such as social and economic conditions, that affect biological maturity [12].

In developed countries such as the US, poverty increases risk of obesity, particularly in women; it is hypothesized that 
this association may be due to factors such as limited access to high quality, inexpensive food, or perceived discrimination over the life course [13]. Neighborhood-level poverty at the census tract level is one contextual factor that can be used to capture socioeconomic status (SES) over time and in a variety of populations including in children who have little control over their own socioeconomic standing [14]. While neighborhood-level poverty (measured in childhood) is associated with obesity in the transition from adolescence to young adulthood [15] and currently living in a poor area is associated with obesity in pregnancy [16], to our knowledge, no studies have examined associations of neighborhood-level poverty at time of menarche with prepregnancy obesity. A life-course approach to studying obesity that focuses on different developmental stages and exposures during those stages [17] may be especially relevant in the study of obesity in nonwhite groups [18]. Thus, we examined if neighborhood-level poverty at the time of menarche was associated with prepregnancy obesity in a sample of pregnant, African-American women.

\section{Methods}

2.1. Sample. The study population and data collection procedures have been described in detail elsewhere [19, 20]. A total of 203 pregnant African-American women in their 2nd trimester being seen for prenatal care at Henry Ford Health System in metropolitan Detroit, MI, were recruited for a study visit. Women provided written informed consent and the study was approved by the Institutional Review Boards at the participating institutions.

2.2. Prepregnancy BMI. As part of routine prenatal care, women were asked to self-report their height and weight from the time just before they became pregnant. Prepregnancy body mass index (BMI, in $\mathrm{kg} / \mathrm{m}^{2}$ ) was calculated. Prepregnancy obesity was defined as BMI $\geq 30$, overweight as BMI $\geq 25$ and $<30$, normal weight as $\mathrm{BMI}>18.5$ and $<25$, and underweight as $\mathrm{BMI} \leq 18.5$; only 8 women were classified as underweight and thus were combined with the normal weight category for analysis. Consistent with other studies [21], selfreported prepregnancy BMI was highly correlated $(r=0.97$; $P<0.001)$ with the 1st measured BMI during pregnancy obtained from the prenatal medical record (measured at mean $9.4 \pm 3.7$ weeks of gestation).

2.3. Menarche and Neighborhood-Level Poverty. Age at menarche was self-reported and time since menarche calculated as the difference between age at study visit and age at menarche. Women were asked to think about when they got their first period and then they were asked to report the address where they were living at the time of menarche. If women were unable to report an address, they were asked if they could report what the major cross streets were where they lived. A few participants reported intersections $(n=7)$, and those were used to identify the closest postal address. We used participant self-reported year of menarche to identify the appropriate census year to obtain neighborhood-level poverty. One participant began menarche in the late 1970 s and her address was mapped to the 1980 census tract; participants reporting menarche between 1980 and 1985 were also mapped to 1980 census tracts. Those who reported menarche between 1986 and 1995 were mapped to 1990 census tracts. Finally, we mapped participants reporting menarche in 1996 and later to 2000 census tracts. Since census tract boundaries may change over time, we used the geocoded address at menarche to obtain data for the appropriate census tract corresponding to the year of census as described above. A similar approach to defining poverty during puberty in a study of premenopausal women was used elsewhere [22].

Neighborhood-level poverty was defined as the proportion of residents in a census tract living below the federal poverty level (using poverty level at the time of the corresponding census). In our sample, at menarche, participants lived in neighborhoods that ranged from having 3.4\% of residents living under the federal poverty level to having $63.1 \%$ of residents living under the federal poverty level. Mooney et al. recently demonstrated that estimates of the association of neighborhood contextual factors, based on census level variables, on health outcomes may result in biased estimates if the continuously distributed factor is used [23]. However, use of quantiles to partition the contextual factor results in unbiased estimates [23]; thus, we calculated sample quartiles of neighborhood-level poverty at the time of menarche. In our sample at menarche, quartile 1 consisted of women living in neighborhoods where the proportion of residents under the federal poverty level ranged from 3.4 to 14\%; Quartile 2 ranged from 14 to $29 \%$; Quartile 3 ranged from 29 to $38 \%$; and quartile 4 consisted of women living in neighborhoods where at least $38 \%$ of residents lived under the federal poverty level.

Women also reported current residential address. We utilized a similar approach to obtain current neighborhood-level poverty. Addresses were mapped to the US Census Bureau's American Community Survey 2006-2010 data to obtain census tract and the corresponding census tract poverty level.

Further, in order to assess individual-level SES at the time of menarche, participants were asked to report the maximum level of education of their mother; to differentiate this from the participant's education, this is referred to as grandmother's education.

2.4. Covariates. Women self-reported race, marital status, and household income. Parity (number of previous viable pregnancies) was obtained from the maternal medical record.

2.5. Statistical Analysis. All analyses were conducted using SAS 9.4. Participant characteristics were compared by prepregnancy BMI category using chi-square or Fisher's exact test for categorical variables and ANOVA or Kruskal-Wallis for continuous variables. Cumulative logistic regression was used to examine the association of neighborhood-level poverty at menarche in quartiles with categorical prepregnancy BMI. Models were fit unadjusted (model I), adjusted for maternal age, marital status, and maternal education at the time of pregnancy (model II), and finally additionally adjusted for parity (model III).

We conducted several sensitivity analyses. First, childhood SES is often associated with adulthood SES. Models 
TABLE 1: Participant characteristics by prepregnancy BMI category $(N=144)$; data are mean \pm standard deviation or $n(\%)$.

\begin{tabular}{|c|c|c|c|c|}
\hline Characteristic & Under/normal weight & Overweight & Obese & $P$ \\
\hline$n$ & $50(34.7 \%)$ & $35(24.3 \%)$ & $59(41.0 \%)$ & \\
\hline Age at menarche (years) & $12.7 \pm 1.6$ & $12.2 \pm 2.0$ & $12.0 \pm 1.7$ & 0.111 \\
\hline Time since menarche (years) & $10.9 \pm 5.0$ & $13.9 \pm 5.9$ & $17.4 \pm 5.5$ & $<0.001$ \\
\hline Age at study visit (years) & $23.6 \pm 4.7$ & $26.1 \pm 5.8$ & $29.4 \pm 5.6$ & $<0.001$ \\
\hline Married/living as married & $8(16.0 \%)$ & $8(22.9 \%)$ & $23(39.0 \%)$ & 0.022 \\
\hline Maternal education (years) & $12.6 \pm 1.4$ & $13.3 \pm 1.9$ & $13.0 \pm 1.7$ & 0.128 \\
\hline Parity & $0.4 \pm 0.6$ & $0.9 \pm 1.2$ & $1.3 \pm 1.2$ & $<0.001$ \\
\hline Nulliparous & $32(64.0 \%)$ & $19(54.3 \%)$ & $17(28.8 \%)$ & 0.001 \\
\hline Grandmother's education (years) $^{\mathrm{a}}$ & $13.3 \pm 2.0$ & $12.7 \pm 2.4$ & $13.2 \pm 2.1$ & 0.700 \\
\hline Neighborhood-level poverty at menarche (\%) & $24 \pm 13$ & $26 \pm 14$ & $30 \pm 13$ & 0.054 \\
\hline Quartiles of neighborhood-level poverty at menarche $\mathrm{b}^{\mathrm{b}}$ & & & & 0.320 \\
\hline Quartile 1 & $16(32.0 \%)$ & $9(26.5 \%)$ & $10(17.0 \%)$ & \\
\hline Quartile 2 & $12(24.0 \%)$ & $11(32.4 \%)$ & $13(22.0 \%)$ & \\
\hline Quartile 3 & $13(26.0 \%)$ & $7(20.6 \%)$ & $16(27.1 \%)$ & \\
\hline Quartile 4 & $9(18.0 \%)$ & $7(20.6 \%)$ & $20(33.9 \%)$ & \\
\hline Current neighborhood-level poverty (\%) & $27 \pm 14$ & $25 \pm 15$ & $31 \pm 15$ & 0.170 \\
\hline Quartiles of current neighborhood-level poverty ${ }^{c}$ & & & & 0.639 \\
\hline Quartile 1 & $12(24.0 \%)$ & $11(32.4 \%)$ & $12(20.7 \%)$ & \\
\hline Quartile 2 & $16(32.0 \%)$ & $8(23.5 \%)$ & $12(20.7 \%)$ & \\
\hline Quartile 3 & $12(24.0 \%)$ & $7(20.6 \%)$ & $18(31.0 \%)$ & \\
\hline Quartile 4 & $10(20.0 \%)$ & $8(23.5 \%)$ & $16(27.6 \%)$ & \\
\hline
\end{tabular}

${ }^{\mathrm{a}} 8$ women missing grandmother's education.

${ }^{\mathrm{b}}$ Quartile 1: 3.4-14\%; Quartile 2: $\geq 14-29 \%$; Quartile 3: $\geq 29-38 \%$; Quartile 4: $\geq 38 \%$.

${ }^{c}$ Quartile 1: 0.8-14\%; Quartile 2: $\geq 14-29 \%$; Quartile 3: $\geq 29-40 \%$; Quartile 4: $\geq 40 \%$.

II and III were fit additionally adjusted for current neighborhood-level poverty quartiles. Second, individual-level SES at the time of menarche may be a potential confounding variable. A previous study found maternal education to be more strongly associated with obesity than paternal education [24]; thus, we utilized grandmother's education as our measure of individual-level childhood SES. Several women $(n=8)$ had missing data for grandmother's education; to preserve sample size for the sensitivity analysis, mean imputation was used and models II and III were fit additionally adjusted for grandmother's education. Finally, since longer time since menarche may be associated with greater risk for obesity, we refit our final models adjusting for time since menarche instead of maternal age.

\section{Results}

As described previously [19], 3 women were a priori excluded due to prepregnancy morbid obesity preventing accurate assessment of weight with standard scales. Five women missing age at first period and five women reporting an address at menarche outside of Michigan were excluded. Sixteen women reported a complete address at menarche that was not mappable to the appropriate census tract and 30 women reported insufficient address information at menarche to allow for geocoding. Our final analytic sample consisted of 144 (72\%) women able to report a valid residence at menarche. There was minimal clustering by neighborhood with the 144 women residing in 131 neighborhoods at the time of menarche.

There were no significant differences in women with and without reported address at menarche with respect to prepregnancy BMI $(P=0.52)$, age at menarche $(P=0.25)$, or age at study visit $(P=0.91)$.

3.1. Prepregnancy BMI. Overall, mean prepregnancy BMI of women was $29.0 \pm 7.4 \mathrm{~kg} / \mathrm{m}^{2} ; 59(41.0 \%)$ women were obese at the start of pregnancy. Participant characteristics are presented by prepregnancy BMI category in Table 1 . Time since menarche, age at study visit during pregnancy, marital status, parity, and being nulliparous were each statistically significantly associated with prepregnancy BMI category (all $P<0.05$ ). Although obese participants had slightly younger age at menarche than overweight and normal weight participants, this was not statistically significant.

3.2. Neighborhood Poverty. At menarche, participants were living in neighborhoods where, on average, $27 \pm 13 \%$ of residents were living below federal poverty levels, which is consistent with US estimates of childhood poverty between 1980 and 2000 [25]. Neighborhood-level poverty at menarche was statistically significantly and positively associated with parity $(P=0.005)$ and inversely associated with being nulliparous $(P=0.004)$ but was not statistically significantly 
TABLE 2: Association of neighborhood-level poverty (in quartiles) at menarche with body mass index category.

\begin{tabular}{|c|c|c|c|c|c|c|}
\hline \multirow{2}{*}{$\begin{array}{l}\text { Neighborhood-level poverty in } \\
\text { quartiles at menarche }\end{array}$} & \multicolumn{2}{|c|}{ Model I } & \multicolumn{2}{|c|}{ Model II } & \multicolumn{2}{|c|}{ Model III } \\
\hline & OR [95\% CI OR] & $P$ & OR [95\% CI OR] & $P$ & OR [95\% CI OR] & $P$ \\
\hline 4 versus 1 & $2.9[1.2,6.9]$ & 0.049 & $2.6[1.0,6.7]$ & 0.048 & $2.3[0.9,6.3]$ & 0.091 \\
\hline 3 versus 1 & $1.7[0.7,4.1]$ & 0.870 & $2.5[1.0,6.7]$ & 0.057 & $1.9[0.7,5.2]$ & 0.214 \\
\hline 2 versus 1 & $1.5[0.6,3.6]$ & 0.752 & $2.5[1.0,6.6]$ & 0.054 & $2.3[0.9,6.0]$ & 0.101 \\
\hline
\end{tabular}

Model I is unadjusted, Model II is adjusted for maternal age, marital status, and maternal education, and Model III is additionally adjusted for parity.

OR, odds ratio; CI, confidence interval.

associated with any other descriptive factor (all $P>0.1$; data not shown).

\subsection{Relationship between Poverty and BMI. Mean neigh-} borhood-level poverty at menarche was higher among women in higher prepregnancy BMI categories (Table 1; $P=$ $0.054)$. Table 2 presents the association of neighborhoodlevel poverty at menarche, in quartiles, with prepregnancy BMI category. In unadjusted models, women in the highest compared to lowest quartiles of neighborhood-level poverty at menarche had statistically significantly increased odds of being in a higher BMI category $(P=0.049)$. After adjusting for maternal age at study visit, maternal education, and marital status, compared to women in the lowest quartile of neighborhood-level poverty at menarche, women in the fourth quartile of poverty had increased odds of being in a higher prepregnancy BMI category compared to women in the 1st quartile $(P=0.048)$. After further adjusting for parity, the association was slightly attenuated and was no longer statistically significant $(P=0.09)$.

3.4. Sensitivity Analysis. There was a statistically significant and positive correlation between neighborhood-level poverty at menarche and current neighborhood-level poverty $(r=$ $0.19, P=0.021)$. In a model adjusted for maternal age, marital status, maternal education, and current neighborhood-level poverty, neighborhood-level poverty at menarche remained statistically significantly associated with prepregnancy obesity $(P=0.048)$; women in the highest compared to lowest quartile of neighborhood-level poverty at menarche had statistically significantly increased odds $(\mathrm{OR}=2.7 ; 95 \% \mathrm{CI}$ $1.0,7.1)$ of being in a higher BMI category. This was slightly attenuated after further adjusting for parity $(\mathrm{OR}=2.5 ; 95 \%$ CI $0.9,7.0 ; P=0.078)$. In a model adjusting for maternal age, maternal education, marital status, and grandmother's education [i.e., measure of childhood SES], the association between neighborhood-level poverty and prepregnancy BMI category was slightly attenuated $(\mathrm{OR}=2.6$; 95\% CI 1.0, 6.8; $P=0.057)$. Further adjusting for parity, the association between neighborhood-level poverty and prepregnancy BMI category was slightly attenuated $(\mathrm{OR}=2.4$; 95\% CI 0.9, 6.6; $P=0.092)$. Finally, in a model adjusting for time since menarche, marital status, and maternal education, the association of neighborhood-level poverty was slightly attenuated $(\mathrm{OR}=2.3 ; 95 \% \mathrm{CI}: 0.9,5.9 ; P=0.096)$ and remained elevated; however it was nonsignificant after adjusting for parity (OR $=2.1 ; 95 \%$ CI: $0.8,5.7 ; P=0.145)$.

\section{Discussion}

We provide first-time, observational evidence suggesting that living in a higher poverty neighborhood at the time of menarche is associated with greater prepregnancy obesity risk in African-American women. The association between neighborhood-level poverty at menarche and prepregnancy obesity remained elevated, although it was no longer statistically significant, after adjusting for parity. Our findings are consistent with a recent study showing lower neighborhood SES at puberty, but not in earlier childhood, was associated with lower levels of sex hormone binding globulin in a sample of 143 premenopausal, nonpregnant women (mean age $36.8 \pm 5.5$ years; $32.2 \%$ African-American) [22]; sex hormone binding globulin levels increase during pregnancy, with lower levels associated with measures of obesity [26].

Neighborhood poverty may impact obesity in several ways [27]. Poorer neighborhoods tend to have lower quality food retailers (i.e., more convenience and liquor stores and/or fast food restaurants) which may promote, by necessity, poorer eating habits [28]. This is often coupled with limited access to safe avenues for physical activity [29]. AfricanAmerican women in particular may adopt unhealthy behaviors in early life, especially overeating of "comfort foods," as a learned, coping strategy to manage chronic stress [30]. Together, along with increased exposure to crime and other stressors, these factors may be contributing to prepregnancy obesity. Such exposures may be especially relevant during puberty [31]. In mouse models, stress (shipping) specifically experienced during puberty results in altered behavioral response to hormones and changes in the hypothalamicpituitary adrenal (HPA) axis response [32]; dysregulation of the HPA axis activity is associated with obesity [33] and thus provides a potential biologic mechanism linking neighborhood poverty at menarche with prepregnancy obesity. Whether this is mediated through HPA axis activity, however, would require future study.

In our sample of African-American women, $41.0 \%$ were obese prepregnancy; this is higher than prepregnancy obesity rates in African-American women (31.5\%) from 9 states in the Pregnancy Risk Assessment Monitoring System (PRAMS) [4]. In contrast, only $20.5 \%$ of Caucasian women were obese before pregnancy in PRAMS [4]. Data from Michigan, where obesity rates are nearly $37 \%$ among African-American adults [34], was not included in PRAMS which may explain why obesity rates are higher in our study.

In a recent study, the disparity in obesity rates between African-American and Caucasian youth was explained by 
neighborhood economic deprivation [27]. Neighborhoodlevel factors in adolescence, such as poverty, could directly promote disparities in prepregnancy obesity seen in adult women in the US. Our analysis focused on neighborhood at the time of menarche; although this is a critical stage in reproductive development [9] and thus may have particular relevance for health during pregnancy, future studies should capture other early-life time points (e.g., neighborhood at birth, at age 18 years) to establish and test the life-course approach.

After adjusting for parity, the association between neighborhood-level poverty and prepregnancy obesity was attenuated and no longer statistically significant. Higher poverty is associated with higher pregnancy rates [35]. Higher parity is also associated with increased prepregnancy obesity risk [4], as weight from previous pregnancies is often maintained into subsequent pregnancies. Because parity may be an intermediate variable in the association of neighborhood-level poverty at menarche with prepregnancy BMI, adjusting for it in our final models may have led to an overadjustment bias [36]. Similarly, the association between neighborhood poverty at menarche and obesity was attenuated when we adjusted for time since menarche instead of maternal age. Earlier age at menarche is associated with adult obesity [12] as well as with growing up in poverty [37] and thus future studies are needed to determine whether earlier age at menarche may act as a confounder or intermediate variable in the association of neighborhood poverty at menarche with prepregnancy obesity.

In the US, over half (51\%) of pregnancies are unintended [38], making preconception programs to reduce the burden of maternal obesity during pregnancy challenging. Further, black women in the US have the highest rate of unintended pregnancy (69\% of pregnancies) [38]. A recent Cochrane systematic review concluded that there were no randomized controlled trials that evaluated the impact of preconception interventions in overweight and obesity [39]. If the risk for prepregnancy obesity originates during puberty, the ideal time to intervene may be during adolescence. However, during pediatric well-child visits, pubertal topics are addressed less frequently than recommended [40] and may represent a gap in care that could improve future maternal and child health.

There are several limitations. Given the observational nature of the study, we cannot provide causal evidence of a relationship between neighborhood poverty at menarche and prepregnancy obesity. The association between neighborhood-level poverty at menarche and prepregnancy obesity, although only slightly attenuated, was not robust to inclusion of parity. Future studies with a larger sample size are needed to better understanding if parity is a confounding factor or a mediating variable. A number of women were unable to report address at menarche; while we found no differences between women who were and were not able to report address at menarche in selected characteristics, our results may be biased by this missing data. As done elsewhere [8], our primary outcome variable was based on self-reported prepregnancy weight. Although there was high correlation between first measured pregnancy weight and self-reported prepregnancy weight in our study and others have shown that categorization of prepregnancy weight comparing self-report to first measured pregnancy weight is similar [41], our results may still be subject to self-report bias. We did not have measures of body size at menarche, so we were unable to account for potential mediating effects via menarche body size. We assigned neighborhood poverty to women based on the decennial census year closest to year of menarche; this may not fully represent the neighborhood condition at menarche and may have introduced increased variability in the exposure assessment. While women's ability to recall age at menarche has been shown to be valid [42], the ability to recall residence at menarche has not been explored; thus we may be subject to recall bias. However, because menarche is a central, unique event of puberty, memory of characteristics surrounding this event (i.e., "flashbulb memories") may have enhanced recall of residential location at this time [42]. Any misclassification due to reporting errors in address at menarche would most likely be nondifferential with respect to prepregnancy weight and thus would bias results toward the null.

Despite these potential limitations, there are a number of strengths of the current study, including the fact that we have a relatively large sample of African-American women, a group at disproportionate risk of prepregnancy obesity [4] and typically underrepresented in research studies. Although measures of individual-level poverty at menarche and during pregnancy are potential confounders, the difficultly in selfreporting family poverty at menarche (compared to reporting a parent's education level) and the lack of willingness of research participants to report current income prevented accounting for these factors in our models. However, we were able to adjust our models for both individual-level SES at menarche (grandmother's education) and at pregnancy (participant's education) and results were similar. To our knowledge, this is the first study to examine neighborhood characteristics at menarche with prepregnancy obesity, which provides a life-course approach linking critical reproductive time points. Future studies are needed to examine such a lifecourse approach; such studies should capture not only information on the neighborhood at critical points in development, but also individual-level characteristics such as BMI and family income over the life course.

\section{Conclusions}

In summary, we found new evidence that neighborhoodlevel poverty at menarche is associated with prepregnancy obesity in African-American women. While interventions in adulthood to reduce preconception BMI have shown success [43], intervening earlier in life during adolescence to promote healthy weight throughout a woman's reproductive years may further improve maternal preconception health [44-46]. Including a social perspective in such interventions, such as the impact of neighborhood-level poverty during adolescence, may be promising [47]. This may be especially important for African-American adolescents, who are at highest risk for living in poverty during childhood [48]. Future studies, however, are needed to confirm our findings and to 
better understand the role of exposures at menarche in health before and during pregnancy.

\section{Competing Interests}

The authors declare that they have no competing interests.

\section{Acknowledgments}

This study was funded by the Institute for Population Sciences, Health Assessment, Administration, Services, and Economics (INPHAASE).

\section{References}

[1] R. Gaillard, B. Durmuş, A. Hofman, J. P. MacKenbach, E. A. P. Steegers, and V. W. V. Jaddoe, "Risk factors and outcomes of maternal obesity and excessive weight gain during pregnancy," Obesity, vol. 21, no. 5, pp. 1046-1055, 2013.

[2] Z. Yu, S. Han, J. Zhu, X. Sun, C. Ji, and X. Guo, "Pre-pregnancy body mass index in relation to infant birth weight and offspring overweight/obesity: a systematic review and meta-analysis," PLoS ONE, vol. 8, no. 4, Article ID e61627, 2013.

[3] S. C. Fisher, S. Y. Kim, A. J. Sharma, R. Rochat, and B. Morrow, "Is obesity still increasing among pregnant women? Prepregnancy obesity trends in 20 states, 2003-2009," Preventive Medicine, vol. 56, no. 6, pp. 372-378, 2013.

[4] S. Y. Kim, P. M. Dietz, L. England, B. Morrow, and W. M. Callaghan, "Trends in pre-pregnancy obesity in nine states, 1993-2003," Obesity, vol. 15, no. 4, pp. 986-993, 2007.

[5] C. Y. W. Lee and G. Koren, "Maternal obesity: effects on pregnancy and the role of pre-conception counselling," Journal of Obstetrics and Gynaecology, vol. 30, no. 2, pp. 101-106, 2010.

[6] N. S. The, C. Suchindran, K. E. North, B. M. Popkin, and P. Gordon-Larsen, "Association of adolescent obesity with risk of severe obesity in adulthood," The Journal of the American Medical Association, vol. 304, no. 18, pp. 2042-2047, 2010.

[7] K. Hollingsworth, L. Callaway, M. Duhig, S. Matheson, and J. Scott, "The association between maltreatment in childhood and pre-pregnancy obesity in women attending an antenatal clinic in Australia," PLoS ONE, vol. 7, no. 12, Article ID e51868, 2012.

[8] J. C. Diesel, L. M. Bodnar, N. L. Day, and C. A. Larkby, "Childhood maltreatment and the risk of pre-pregnancy obesity and excessive gestational weight gain," Maternal and Child Nutrition, 2014.

[9] D. F. Abetew, D. A. Enquobahrie, M. Dishi, C. B. Rudra, R. S. Miller, and M. A. Williams, "Age at menarche, menstrual characteristics, and risk of preeclampsia," ISRN Obstetrics and Gynecology, vol. 2011, Article ID 472083, 6 pages, 2011.

[10] C. Currie, N. Ahluwalia, E. Godeau, S. Nic Gabhainn, P. Due, and D. B. Currie, "Is obesity at individual and national level associated with lower age at menarche? Evidence from 34 countries in the health behaviour in school-aged children study," Journal of Adolescent Health, vol. 50, no. 6, pp. 621-626, 2012.

[11] P. Prentice and R. M. Viner, "Pubertal timing and adult obesity and cardiometabolic risk in women and men: a systematic review and meta-analysis," International Journal of Obesity, vol. 37, no. 8, pp. 1036-1043, 2013.

[12] M. B. Pierce and D. A. Leon, "Age at menarche and adult BMI in the Aberdeen Children of the 1950s Cohort Study," American Journal of Clinical Nutrition, vol. 82, no. 4, pp. 733-739, 2005.
[13] D. J. Hruschka, "Do economic constraints on food choice make people fat? A critical review of two hypotheses for the povertyobesity paradox," American Journal of Human Biology, vol. 24, no. 3, pp. 277-285, 2012.

[14] N. Krieger, J. T. Chen, P. D. Waterman, D. H. Rehkopf, and S. V. Subramanian, "Painting a truer picture of US socioeconomic and racial/ethnic health inequalities: the public health disparities geocoding project," American Journal of Public Health, vol. 95, no. 2, pp. 312-323, 2005.

[15] H. Lee, K. M. Harris, and P. Gordon-Larsen, "Life course perspectives on the links between poverty and obesity during the transition to young adulthood," Population Research and Policy Review, vol. 28, no. 4, pp. 505-532, 2009.

[16] E. Sellström, G. Arnoldsson, M. Alricsson, and A. Hjern, “Obesity prevalence in a cohort of women in early pregnancy from a neighbourhood perspective," BMC Pregnancy and Childbirth, vol. 9, article 37, 2009.

[17] M. Novak, C. Ahlgren, and A. Hammarström, "A life-course approach in explaining social inequity in obesity among young adult men and women," International Journal of Obesity, vol. 30, no. 1, pp. 191-200, 2006.

[18] G. Wahi and S. S. Anand, "Race/ethnicity, obesity, and related cardio-metabolic risk factors: a life-course perspective," Current Cardiovascular Risk Reports, vol. 7, no. 5, pp. 326-335, 2013.

[19] A. E. Cassidy-Bushrow, R. M. Peters, D. A. Johnson, J. Li, and D. S. Rao, "Vitamin d nutritional status and antenatal depressive symptoms in African American women," Journal of Women's Health, vol. 21, no. 11, pp. 1189-1195, 2012.

[20] M. J. Wilusz, R. M. Peters, and A. E. Cassidy-Bushrow, "Course of depressive symptoms across pregnancy in african american women," Journal of Midwifery and Women's Health, vol. 59, no. 4, pp. 411-416, 2014.

[21] D. Shin, H. Chung, L. Weatherspoon, and W. O. Song, "Validity of prepregnancy weight status estimated from self-reported height and weight," Maternal and Child Health Journal, vol. 18, no. 7, pp. 1667-1674, 2014.

[22] M. E. Bleil, B. M. Appelhans, M. D. Latham et al., "Neighborhood socioeconomic status during childhood versus puberty in relation to endogenous sex hormone levels in adult women," Nursing Research, vol. 64, no. 3, pp. 211-220, 2015.

[23] S. J. Mooney, C. A. Richards, and A. G. Rundle, “There goes the neighborhood effect: bias owing to nondifferential measurement error in the construction of neighborhood contextual measures," Epidemiology, vol. 25, no. 4, pp. 528-535, 2014.

[24] A. Lamerz, J. Kuepper-Nybelen, C. Wehle et al., "Social class, parental education, and obesity prevalence in a study of sixyear-old children in Germany," International Journal of Obesity and Related Metabolic Disorders, vol. 29, no. 4, pp. 373-380, 2005.

[25] P. Whiteford and W. Adema, "What works best in reducing child poverty: a benefit or work strategy?" OECD Social, Employment and Migration Working Papers No 51, 2007, http://www .oecd.org/social/family/38227981.pdf.

[26] E. A. M. Kuijper, J. C. F. Ket, M. R. Caanen, and C. B. Lambalk, "Reproductive hormone concentrations in pregnancy and neonates: a systematic review," Reproductive BioMedicine Online, vol. 27, no. 1, pp. 33-63, 2013.

[27] L. M. Rossen, "Neighbourhood economic deprivation explains racial/ethnic disparities in overweight and obesity among children and adolescents in the USA," Journal of Epidemiology and Community Health, vol. 68, no. 2, pp. 123-129, 2014. 
[28] S. N. Zenk, A. J. Schulz, and A. M. Odoms-Young, "How neighborhood environments contribute to obesity," The American Journal of Nursing, vol. 109, no. 7, pp. 61-64, 2009.

[29] I. A. Corral, H. Landrine, Y. Hao, L. Zhao, J. L. Mellerson, and D. L. Cooper, "Residential segregation, health behavior and overweight/obesity among a national sample of African American adults," Journal of Health Psychology, vol. 17, no. 3, pp. 371-378, 2012.

[30] J. S. Jackson, K. M. Knight, and J. A. Rafferty, "Race and unhealthy behaviors: chronic stress, the HPA Axis, and physical and mental health disparities over the life course," American Journal of Public Health, vol. 100, no. 5, pp. 933-939, 2010.

[31] J. E. Schneider, J. M. Brozek, and E. Keen-Rhinehart, "Our stolen figures: the interface of sexual differentiation, endocrine disruptors, maternal programming, and energy balance," Hormones and Behavior, vol. 66, no. 1, pp. 104-119, 2014.

[32] J. Laroche, L. Gasbarro, J. P. Herman, and J. D. Blaustein, "Reduced behavioral response to gonadal hormones in mice shipped during the peripubertal/adolescent period," Endocrinology, vol. 150, no. 5, pp. 2351-2358, 2009.

[33] A. C. Incollingo Rodriguez, E. S. Epel, M. L. White, E. C. Standen, J. R. Seckl, and A. J. Tomiyama, "Hypothalamicpituitary-adrenal axis dysregulation and cortisol activity in obesity: a systematic review," Psychoneuroendocrinology, vol. 62, pp. 301-318, 2015.

[34] Centers for Disease Control and Prevention, Division of Nutrition, Physical Activity, and Obesity. Prevalence of SelfReported Obesity Among Non-Hispanic Black Adults by State and Territory, BRFSS, 2012-2014, 2015, http://www.cdc.gov/ obesity/data/table-non-hispanic-black.html.

[35] L. B. Finer and M. R. Zolna, "Unintended pregnancy in the United States: incidence and disparities, 2006," Contraception, vol. 84, no. 5, pp. 478-485, 2011.

[36] M. A. Brookhart, T. Stürmer, R. J. Glynn, J. Rassen, and S. Schneeweiss, "Confounding control in healthcare database research: challenges and potential approaches," Medical Care, vol. 48, no. 6, pp. S114-S120, 2010.

[37] A. F. Jorm, H. Christensen, B. Rodgers, P. A. Jacomb, and S. Easteal, "Association of adverse childhood experiences, age of menarche, and adult reproductive behavior: does the androgen receptor gene play a role?" American Journal of Medical Genetics, Part B-Neuropsychiatric Genetics, vol. 125, no. 1, pp. 105-111, 2004.

[38] L. B. Finer and M. R. Zolna, "Shifts in intended and unintended pregnancies in the United States, 2001-2008," American Journal of Public Health, vol. 104, no. 1, pp. S43-S48, 2014.

[39] N. Opray, R. M. Grivell, A. R. Deussen et al., "Directed preconception health programs and interventions for improving pregnancy outcomes for women who are overweight or obese," Cochrane Database of Systematic Reviews, vol. 7, Article ID CD010932, 2015.

[40] C. Norlin, M. A. Crawford, C. T. Bell, X. Sheng, and M. T. Stein, "Delivery of well-child care: a look inside the door," Academic Pediatrics, vol. 11, no. 1, pp. 18-26, 2011.

[41] E. Holland, T. A. M. Simas, D. K. D. Curiale, X. Liao, and M. E. Waring, "Self-reported pre-pregnancy weight versus weight measured at first prenatal visit: effects on categorization of pre-pregnancy body mass index," Maternal and Child Health Journal, vol. 17, no. 10, pp. 1872-1878, 2013.

[42] A. Must, S. M. Phillips, E. N. Naumova et al., "Recall of early menstrual history and menarcheal body size: after 30 years, how well do women remember?" American Journal of Epidemiology, vol. 155, no. 7, pp. 672-679, 2002.

[43] C. S. Weisman, M. M. Hillemeier, D. S. Downs et al., "Improving women's preconceptional health: long-term effects of the strong healthy women behavior change intervention in the Central Pennsylvania Women's health study," Women's Health Issues, vol. 21, no. 4, pp. 265-271, 2011.

[44] A. M. Siega-Riz and J. C. King, "Position of the American Dietetic Association and American Society for Nutrition: obesity, reproduction, and pregnancy outcomes," Journal of the American Dietetic Association, vol. 109, no. 5, pp. 918-927, 2009.

[45] S. B. Procter and C. G. Campbell, "Position of the academy of nutrition and dietetics: nutrition and lifestyle for a healthy pregnancy outcome," Journal of the Academy of Nutrition and Dietetics, vol. 114, no. 7, pp. 1099-1103, 2014.

[46] L. L. Kaiser and C. G. Campbell, "Practice paper of the Academy Of Nutrition And Dietetics abstract: nutrition and lifestyle for a healthy pregnancy outcome," Journal of the Academy of Nutrition and Dietetics, vol. 114, no. 9, article 1447, 2014.

[47] G. Sutherland, S. Brown, and J. Yelland, "Applying a social disparities lens to obesity in pregnancy to inform efforts to intervene," Midwifery, vol. 29, no. 4, pp. 338-343, 2013.

[48] ASPE Human Services Policy Staff, ASPE ISSUE BRIEF Information on Poverty and Income Statistics: A Summary of 2012 Current Population Survey Data, 2015, http://aspe.hhs.gov/hsp/ 12/povertyAndIncomeEst/ib.cfm. 


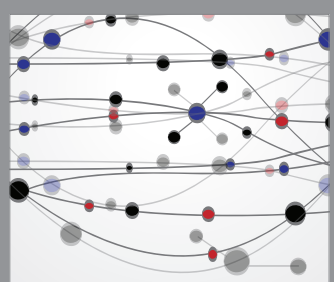

The Scientific World Journal
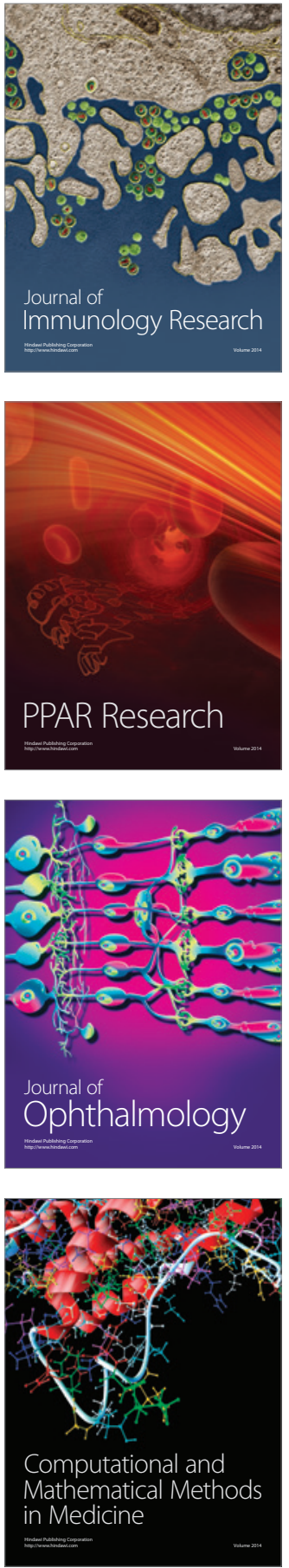

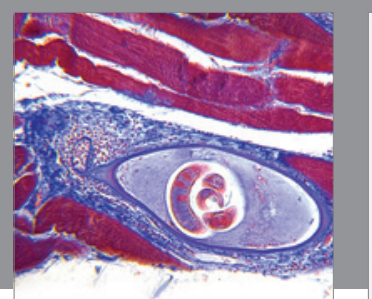

Gastroenterology Research and Practice

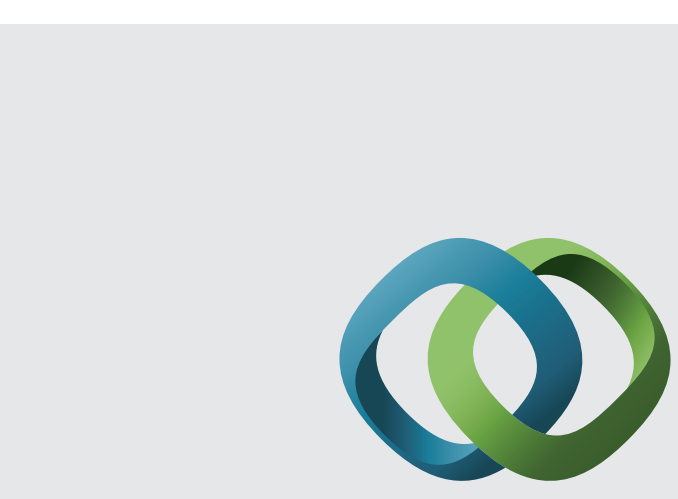

\section{Hindawi}

Submit your manuscripts at

http://www.hindawi.com
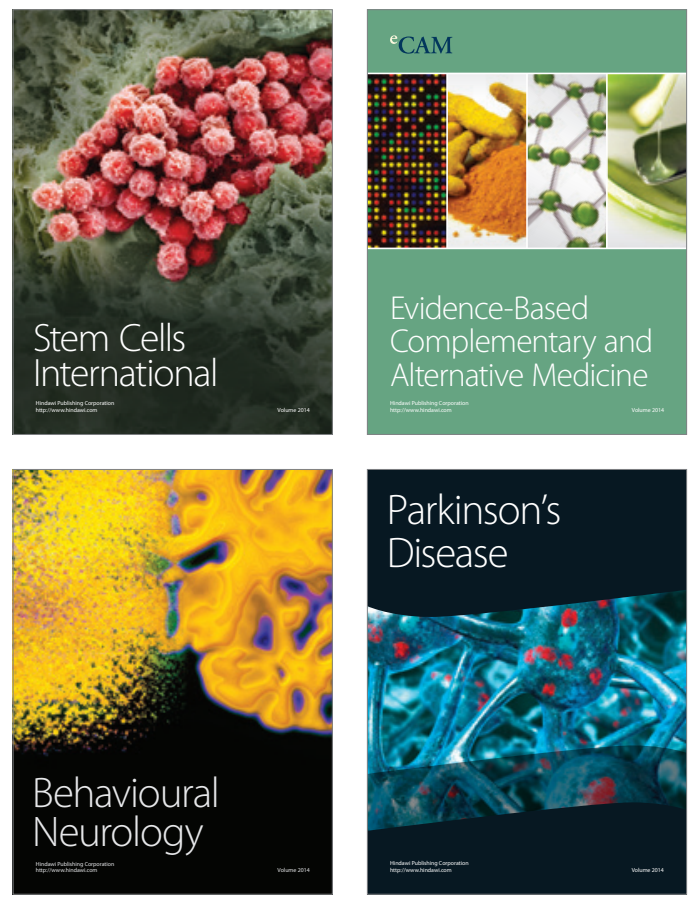
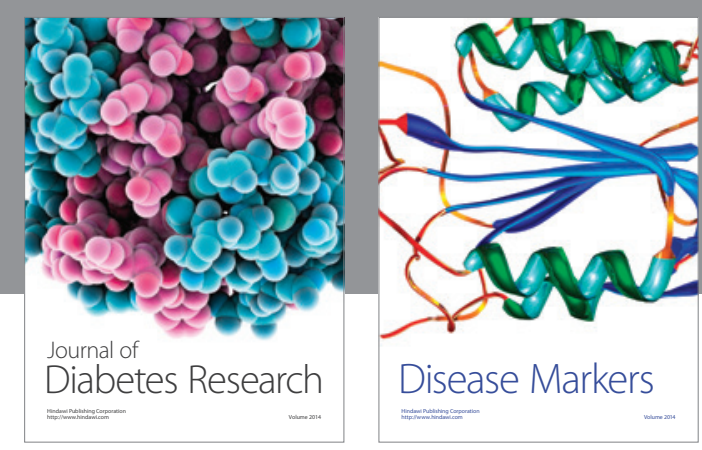

Disease Markers
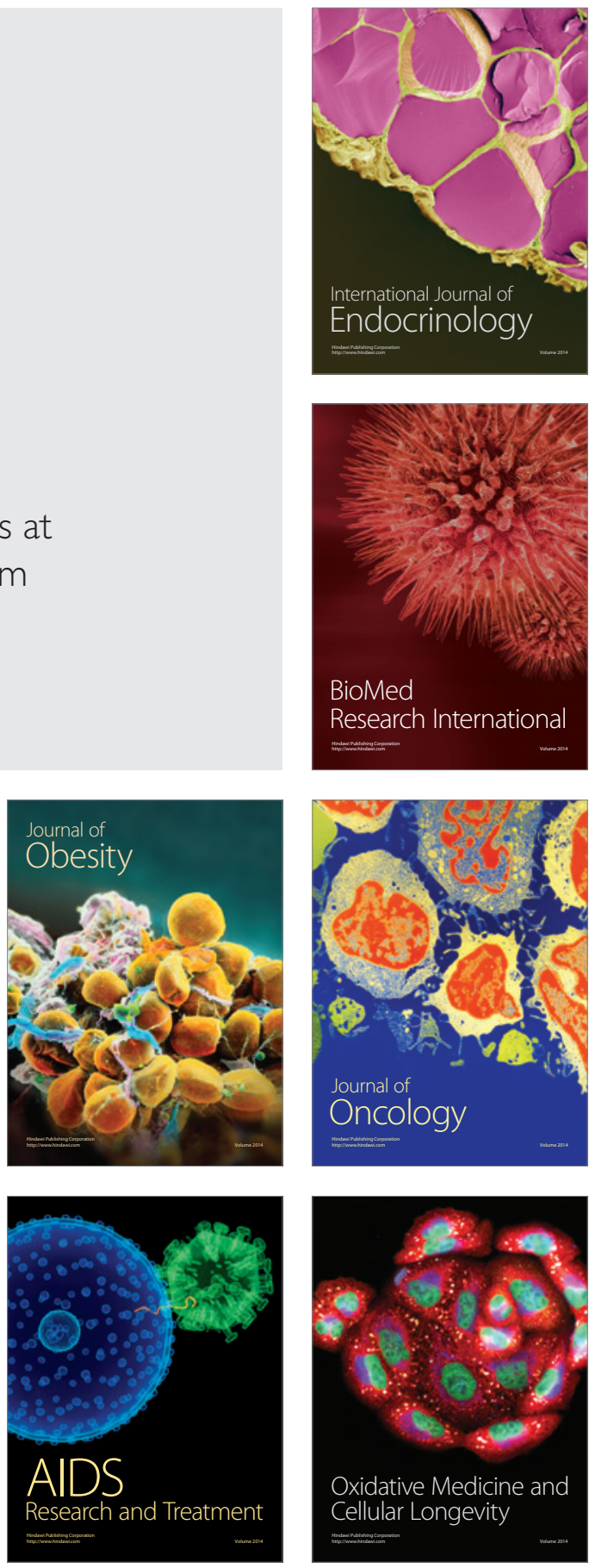